\title{
Computational and experimental study on local ship loads in short and steep waves
}

\author{
Satu K. Hänninen • Tommi Mikkola • \\ Jerzy Matusiak
}

Received: 9 November 2012/Accepted: 14 July 2013/Published online: 13 August 2013

(c) The Author(s) 2013. This article is published with open access at Springerlink.com

\begin{abstract}
Currently, little information exists on the validity of interface-capturing methods in predicting local ship wave loads in short and steep waves. This study compares computational and experimental results in such a case $\left(k A=0.24, L_{\text {wave }} / L_{\text {ship }}=0.16\right)$. The results allow the variation of wave loading between ten locations in the bow area of the ship to be observed. The computations were performed with an unstructured RANS solver that models free-surface flows with a volume-of-fluid method. In the model tests, the wave loads were measured with pressure sensors. The analysis of the results focuses on the wave conditions and on the pressure histories of the local wave loads. The computational and experimental results are in good qualitative agreement and encourage the further use of the computational results.
\end{abstract}

Keywords Local ship wave loads - Short and steep waves $\cdot$ RANS $\cdot$ Interface-capturing methods (VOF) . Model-tests

\section{Introduction}

Interface-capturing methods have an increasing role in predicting ship wave loads at present. One advantage of such methods is that they can give very detailed flow information in both the spatial and time domains. In the case of ship wave loads, the detailed information allows the origin of the loading to be disclosed and supports the

Visiting address of the authors: Tietotie 1A, 02150 Espoo, Finland.

S. K. Hänninen $(\bowtie) \cdot T$. Mikkola · J. Matusiak

Department of Applied Mechanics, School of Engineering,

Aalto University, PO Box 15300, 00076 Aalto, Finland

e-mail: Satu.Hanninen@aalto.fi optimisation of the hull form. The interface-capturing methods are set apart from more traditional seakeeping methods like potential flow theories, because they can predict arbitrary free-surface behaviour, e.g., wave breaking, and the related loads.

In this study, we are interested in the point-wise pressure histories on the bow of a ship in short and steep waves. This relates to the need to learn more about the origin of the second harmonic springing. Here, the critical waves, which lead to a state of resonance between the wave loading and two-node hull eigenmodes, are very short in comparison to the hull dimensions. In order to have a distinct contribution of higher harmonic loading, the encountered waves have been chosen to be very steep. Such wave conditions can lead to a serious deformation of the free-surface level near the hull, which underlines the importance of using an advanced method for predicting the behaviour of the free-surface level. The main question in this paper is whether the interface-capturing methods are suitable for predicting local ship wave loads in very short and steep waves.

To find out if a method is suitable for predicting a flow case, its results are typically compared with the respective experimental ones. In the case of predicting ship wave loads with interface-capturing methods, several previous studies have contributed to this task, e.g. [1-16]. Even if such studies already exist, it is difficult to reach general conclusions on the validity of these methods. One of the reasons is the large number of possible options regarding the characteristics of both waves and hull forms. This means that publishing more validation-type studies on different ship wave load cases increases the confidence in the methods. Further, the requirements on the modelling can be different, depending on the level of detail on which the wave loads are studied. In practice, there are several 
previous validation-type studies on global ship wave loads, e.g. $[1-3,5,6,8-14,16]$, but the examples of studies on local loads like pressure histories are fewer in number, e.g. $[4,7,12,15]$.

From the theoretical point of view, the interface-capturing methods themselves are not known to set any limitations on the ship wave load cases that are allowed. In this regard, the primary purpose of validation-type studies is to learn if the selected way of applying a method to a flow case is correct. User-made choices, which can seriously affect the solution, are the discretisation resolution (both grid spacing and time steps) and the level of the iterative convergence for each time step. Therefore, studying the effects of these matters is a fundamental part of analysing the numerical results. The level of detail with which the solution behaviour as a function of resolution is studied can vary significantly between showing qualitative solution behaviour and defining quantified numerical uncertainties. As for quantifying the uncertainties for time histories like (impact-type) pressure histories in this study, the scientific literature seems not to have presented practical examples yet. In general, the solution should be in the vicinity of the asymptotic range when quantifying uncertainties. In the case of ship wave loads, reaching the asymptotic range is a challenge even for the lowest harmonic components, although quantified uncertainties have been presented for mean and first harmonic global loads in [5, 6, 14, 16] and for the 1st-3rd harmonic components of the vertical force acting on a ship frame in [17]. The qualitative solution behaviour of ship wave loads has been studied in [2, 4, 10]. As for studying the solution behaviour of local pressure histories, the qualitative approach has been applied in [4].

In this study, we focus on comparing computed and measured local pressure histories on the bow area of a ship in order to study the reliability of the present computational results. The ratio of the present wave and ship length is 0.16 , while the respective ratio has been between 0.60 and 1.09 in the previous studies on local pressure histories. In this study, the behaviour of the numerical results has been observed by repeating the computation with three systematically refined resolutions. One important part of the present study was to conduct specific model tests in order to have the necessary experimental data. In this paper, the flow case is defined in Sect. 2. Then the applied numerical and experimental approaches are described in Sects. 3 and 4 , respectively. The wave conditions are addressed in Sect. 5 and the results with regard to the local wave loads in Sect. 6. Finally, the conclusions are reached in Sect. 7.

This study continues our previous work in [17]. The previous work has focused on quantifying the numerical uncertainty of the loading in terms of the harmonic components of the force distribution in the bow area. As such, the paper [17] focused on the numerical behaviour of the solution only. This paper studies the validity of the flow behaviour predicted by the numerical method. In this study, we have redone the computations and conducted model tests of the flow case. In the new computations, a RANSsolver has been applied in order to include the effect of turbulence. Further, the iterative performance of the numerical solutions has been improved by applying another discretisation scheme for the convective term of the volume fraction conservation equation.

\section{Flow case}

The flow case is a passenger ship advancing in short and steep waves. The ship frames are given in Fig. 1 and the case parameters in Table 1 . We have studied this case and another similar one previously in [17-19].

The ship motions due to the waves are assumed to be very minor, because the ratio of the wave and ship lengths is such small, $L_{\text {wave }} / L_{\text {ship }}=0.16$. Therefore, the computations have been simplified by fixing the position of the ship. The justification of this assumption is studied in Sect. 5

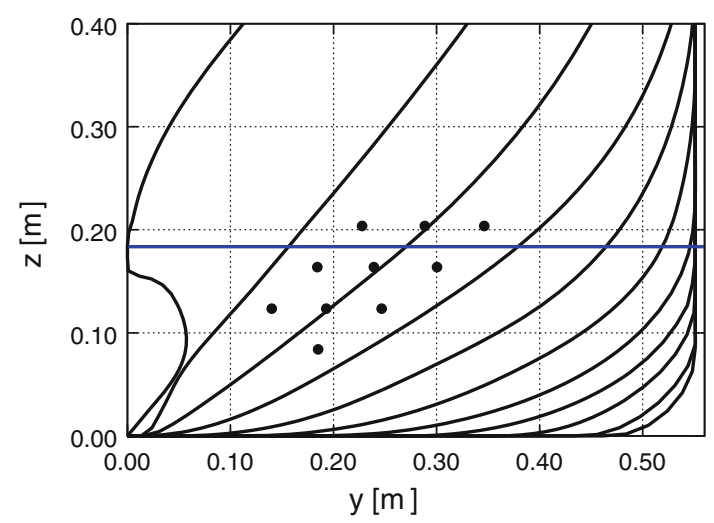

Fig. 1 Ship frames between the fore perpendicular and the midship, locations of the pressure sensors

Table 1 Ship and wave particulars on model scale

\begin{tabular}{llll}
\hline $\begin{array}{l}\text { Length } L_{\text {ship }} \\
\text { Breadth }\end{array}$ & $\begin{array}{l}6.69 \mathrm{~m} \\
1.10 \mathrm{~m}\end{array}$ & $\begin{array}{l}\text { Wave length } L_{\text {wave }} \\
\text { Wave height } \\
H_{\text {wave }}=2 A\end{array}$ & $\begin{array}{l}1.05 \mathrm{~m} \\
0.08 \mathrm{~m}\end{array}$ \\
Draught $^{\mathrm{a}}$ & $0.184 \mathrm{~m}$ & $\begin{array}{c}\text { Wave steepness } k A \\
\text { Block coefficient }\end{array}$ & 0.72 \\
$\begin{array}{l}\text { Velocity } V_{\text {ship }} \\
\text { Froude number }\end{array}$ & $1.47 \mathrm{~m} / \mathrm{s}$ & 0.181 \\
$\begin{array}{l}\text { Reynolds } \\
\text { number }\end{array}$ & $0.982 \times 10^{7}$ & $0.38 \mathrm{~s}$ \\
\hline
\end{tabular}

${ }^{\text {a }}$ At $V_{\text {ship }}=1.47 \mathrm{~m} / \mathrm{s}$ 


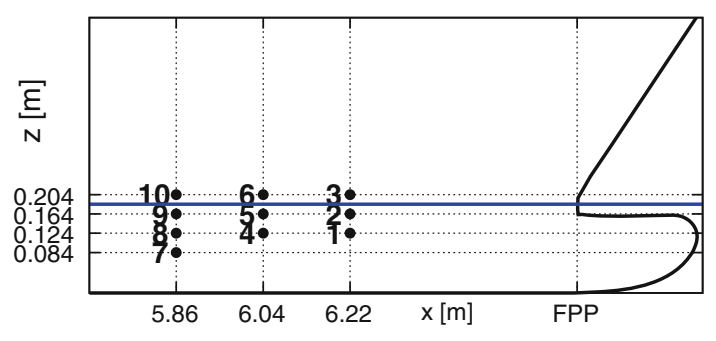

Fig. 2 Pressure sensors and their numbering seen from the starboard side of the hull

based on the measured heave and pitch motions in the model tests.

The results of interest are the characteristics of the waves encountered and the local pressure histories at ten locations in the area of the bow. The locations of the pressure sensors are given in Fig. 2.

\section{Computational approach}

\subsection{Flow solver}

In this study, the computations are performed with an unstructured finite volume solver ISIS-CFD $[20,21]$ that models free-surface flows with a volume-of-fluid method.

The governing equations for mass, momentum, and volume fraction conservation are given in Cartesian coordinates in Eqs. 1-3, respectively. They are written for the incompressible flow of viscous fluid [20]. The mass conservation gains the following form when incompressible phases with constant densities of the distinct phases are considered and when the so-called space conservation law is satisfied in the case of a moving grid [20].

$$
\begin{aligned}
& \int_{S} \vec{U} \cdot \vec{n} \mathrm{~d} S=0, \\
& \frac{\partial}{\partial t} \int_{V(t)} \rho \vec{U} \mathrm{~d} V+\int_{S(t)} \rho \vec{U}\left(\vec{U}-\vec{U}_{d}\right) \cdot \vec{n} \mathrm{~d} S \\
& \quad=\int_{V(t)} \nabla \cdot \vec{T} \mathrm{~d} V-\int_{S(t)} p \vec{n} \mathrm{~d} S+\int_{V(t)} \rho \vec{g} \mathrm{~d} V, \\
& \frac{\partial}{\partial t} \int_{V(t)} c_{i} \mathrm{~d} V+\int_{S(t)} c_{i}\left(\vec{U}-\vec{U}_{d}\right) \cdot \vec{n} \mathrm{~d} S=0,
\end{aligned}
$$

where $V$ is the control volume that is bounded by the closed surface $S . \vec{U}_{d}$ is the velocity of the surface and $\vec{n}$ its outwards-directed unit normal vector. $\vec{U}$ denotes the velocity field, $p$ the pressure field, $\vec{T}$ the stress tensor, $\vec{g}$ the gravity vector, and $c_{i}$ the volume fraction of the fluid $i$. The present flow includes only two fluids: air and water, in other words $i=$ air or $i=$ water. The value $c_{\mathrm{air}}=c_{\mathrm{water}}=1 / 2$ represents the free-surface level.

The fluid density and the fluid viscosity are defined for each control volume with

$$
\begin{aligned}
& \rho=c_{\text {water }} \cdot \rho_{\text {water }}+c_{\text {air }} \cdot \rho_{\text {air }} \text { and } \\
& \mu=c_{\text {water }} \cdot \mu_{\text {water }}+c_{\text {air }} \cdot \mu_{\text {air }},
\end{aligned}
$$

respectively.

The turbulence model applied is Menter's STT $k$ - $\omega$ model with wall functions. Its implementation in the present flow solver is given in [21].

The discretisation of the governing equations is explained in [20]. In the present study, second-order discretisation schemes were chosen for the user-defined options: a second-order backward scheme for the time derivatives [20], the gamma differencing scheme (GDS) for the convective terms of momentum and turbulence equations [20], and the blended reconstructed interface capturing scheme (BRICS) for the convective term of the volume fraction conservation equation [22].

\subsection{Setups for the flow solver}

The present setups for the flow solver are to some extent similar to that described and applied in [17] and applied in $[18,19]$.

The computation was repeated six times in total to study both the effect of the resolution and the effect of the iteration number within a time step. The effects of both the spatial and the temporal resolutions were studied simultaneously with three resolutions. Both the spatial and the temporal resolutions were scaled with the ratios 1.25 (coarse/medium) and 1.20 (medium/fine); see Table 2. The significance of the iterative error was tested on each resolution by repeating the computation with two different iteration numbers (10 and 20 iterations per time step), while the requirement for the decrease of the residual (infinity norms) was set so high that practically it did not limit the iteration number. These present choices on the

Table 2 Information on grids and time steps

\begin{tabular}{llllcl}
\hline & $\begin{array}{l}\text { Number of } \\
\text { cells }\end{array}$ & $t_{\mathrm{e}} / \Delta t^{\mathrm{a}}$ & $L_{\text {wave }} / L_{\mathrm{c}}^{\mathrm{b}}$ & $H_{\text {wave }} / H_{\mathrm{c}}{ }^{\mathrm{c}}$ & $y_{1}{ }^{\mathrm{d}}$ \\
\hline Coarse & $2.29 \mathrm{M}$ & 245.16 & 58.32 & 8.00 & 0.002 \\
Medium & $3.71 \mathrm{M}$ & 306.45 & 72.90 & 10.00 & 0.0016 \\
Fine & $6.53 \mathrm{M}$ & 367.86 & 87.49 & 11.94 & 0.001333 \\
\hline
\end{tabular}

This information has been published previously in [17], apart from that on the thickness of the first layer and on the number of cells

a Time step

b Length of the cells in the refinement boxes $b 1$ and $b 2$

c Height of the cells in the refinement boxes $b 1$ and $b 2$

d Thickness of the first layer in the viscous layer 
Table 3 Locations of the grid boundaries [17]

\begin{tabular}{lrcr}
\hline & Coarse & Medium & Fine \\
\hline$\left|x_{\text {FPP }}-x_{\text {min }}\right| / L_{\text {wave }}$ & 14.70 & 14.70 & 14.70 \\
$\left|x_{\text {FPP }}-x_{\text {max }}\right| / L_{\text {wave }}$ & 2.86 & 2.86 & 2.86 \\
$\left|y_{\text {FPP }}-y_{\text {min }}\right| / L_{\text {wave }}$ & 0.00 & 0.00 & 0.00 \\
$\left|y_{\text {FPP }}-y_{\text {max }}\right| / L_{\text {wave }}$ & 6.63 & 6.63 & 6.63 \\
$\left|z_{\text {FPP }}-z_{\text {min }}\right| / L_{\text {wave }}$ & 7.14 & 8.57 & 7.95 \\
$\left|z_{\text {FPP }}-z_{\text {max }}\right| / L_{\text {wave }}$ & 2.61 & 1.18 & 1.80 \\
\hline
\end{tabular}

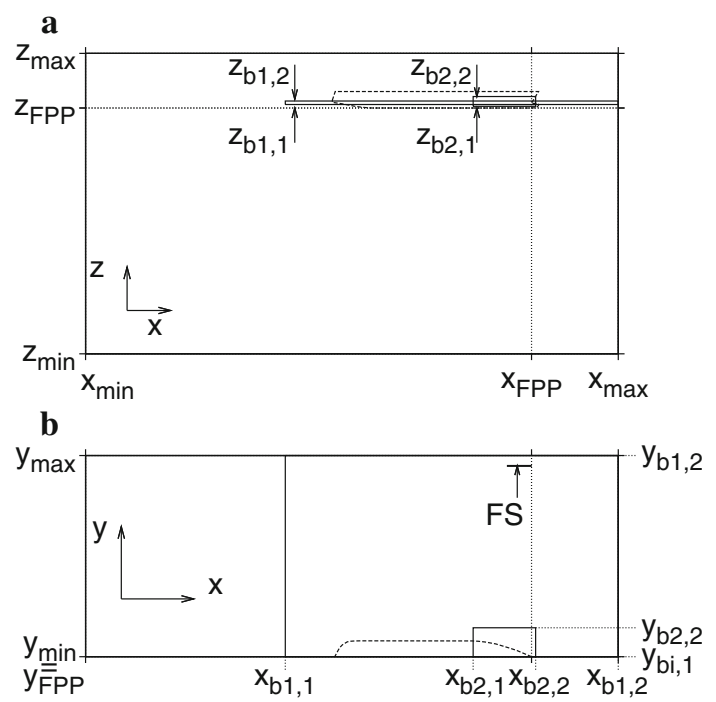

Fig. 3 Coordinate axes, boundaries of the computational domain $\left(x_{\min }, x_{\max }, y_{\min }, y_{\max }, z_{\min }, z_{\max }\right)$, boundaries of the refinement boxes $b 1$ and $b 2\left(x_{b i, 1}, x_{b i, 2}, y_{b i, 1}, y_{b i, 2}, z_{b i, 1}, z_{b i, 2}\right)$, location of the fore perpendicular $\left(x_{\mathrm{FPP}}, y_{\mathrm{FPP}}, z_{\mathrm{FPP}}\right)$ : a $x z$-level b $x y$-level, the line $F S$ indicates the location of the analysis of the behaviour of the waves

Table 4 Boundary conditions

\begin{tabular}{llll}
\hline$x_{\min }$ & Far-field condition & $x_{\max }$ & $\begin{array}{l}\text { Wave generator, first order } \\
\text { Stokes }\end{array}$ \\
$y_{\min }$ & Mirror condition & $y_{\max }$ & $\begin{array}{l}\text { Mirror condition } \\
z_{\min }\end{array}$ \\
$\begin{array}{c}\text { Prescribed } \\
\text { pressure }\end{array}$ & $z_{\max }$ & Prescribed pressure \\
Deck & Slip wall & Hull & Wall with wall-functions \\
\hline
\end{tabular}

iteration within a time step check how the recommendations for the present solver work for the present flow case. According to the recommendations, 20 iterations and the decrease in the residuals by two orders of magnitude should be used for sea-keeping computations [23].

The computational domain includes only one half of the ship hull because of the symmetric flow case. The computational domain moves with the ship. The locations of the domain boundaries are given in Table 3, see also Fig. 3. The locations of the upper and the lower boundaries are slightly different between the three resolutions to
Table 5 Locations of the boundaries of the refinement boxes $b 1$ and b2 [17]

\begin{tabular}{llllll}
\hline & $b 1$ & $b 2$ & & $b 1$ & $b 2$ \\
\hline$\left|x_{\mathrm{FPP}}-x_{b i, 1}\right| / L_{\text {wave }}$ & 8.12 & 1.93 & $\left|x_{\mathrm{FPP}}-x_{b i, 2}\right| / L_{\text {wave }}$ & 2.86 & 0.13 \\
$\left|y_{\mathrm{FPP}}-y_{b i, 1}\right| / L_{\text {wave }}$ & 0.00 & 0.00 & $\left|y_{\mathrm{FPP}}-y_{b i, 2}\right| / L_{\text {wave }}$ & 6.63 & 0.95 \\
$\left|z_{\mathrm{FPP}}-z_{b i, 1}\right| / L_{\text {wave }}$ & 0.12 & 0.06 & $\left|z_{\mathrm{FPP}}-z_{b i, 2}\right| / L_{\text {wave }}$ & 0.23 & 0.38 \\
\hline
\end{tabular}

Table 6 Information on fluids

\begin{tabular}{lcll}
\hline Water density & $998.1 \mathrm{~kg} / \mathrm{m}^{3}$ & Air density & $1.2 \mathrm{~kg} / \mathrm{m}^{3}$ \\
Water viscosity & $0.001 \mathrm{~kg} / \mathrm{sm}$ & Air viscosity & $\begin{array}{c}1.85 \times 10^{-5} \\
\mathrm{~kg} / \mathrm{sm}\end{array}$ \\
$\begin{array}{l}\text { Standard } \\
\text { gravity }\end{array}$ & $9.81 \mathrm{~m} / \mathrm{s}^{2}$ & & \\
\hline
\end{tabular}

ensure that the location of the initial free-surface level coincides with a cell face with each grid. The boundary conditions are given in Table 4. No special treatment is applied at the outlet boundary $\left(x_{\min }\right)$ for wave absorption. The grids were generated with the hexahedral grid generator Hexpress (version 2.11-1).

The spatial domain has three sub-domains of local refinements: one to transport the waves in the computational domain (refinement box $b 1$, Fig. 3; Table 5); another one to refine the domain near the bow in the y-direction (refinement box $b 2$, Fig. 3; Table 5), and a third one near the hull surface for the boundary layer. The cell sizes in the refinement boxes $b 1$ and $b 2$ are given in Table 2 . As for the boundary layer, the first cell heights are given in Table 2 and the stretching ratio is 1.20 . The first cell heights were selected in such a way that the dimensionless distance from the wall $y^{+}$gets the value of roughly 30 in the area of the stern of the ship. The resolutions of the surface grid on the hull are similar as in [17].

At the beginning of the computation (0.00-3.00 s), the ship accelerates according to an acceleration ramp of the form $0.5-0.5 \cos (t \pi / 3.00 \mathrm{~s})$. The wave generation starts at the inlet boundary at the beginning of the simulation. The results to be analysed cover the time frame $6.98-10.80 \mathrm{~s}$, which includes 10 encounter periods.

Table 6 gives information on the fluid properties.

\subsection{Representing the computed results}

The pressure histories are low-pass filtered with the threshold frequency of $30.5 \times$ encounter frequency in order to remove high frequency noise.

Because of the unstructured grids, the computational points are located arbitrarily on the hull and their locations depend on the resolution. In order to have coherently selected observation areas for each sensor at each resolution, all the computational points within a distance of 
$1.33 \times R_{\text {sensor }}$ from a sensor centre are taken into account when estimating the pressure that acts on the surface area of one pressure sensor.

In practice, there are 1-4 computational points within the selected observation area of one sensor depending on the resolution and on the location of the sensor. Further, the computational data includes ten encounter periods. Thus, the computational data include 10-40 encounter periods of point-wise pressures per one sensor per a computation. This number of encounter periods is denoted as $n_{\mathrm{ep}}$ in the following. The pressure history for one sensor is presented as an average encounter period $p(t)$

$p(t)=\frac{1}{n_{\mathrm{ep}}} \sum_{j=1}^{n_{\mathrm{ep}}} p_{j}(t)$.

All the results at the $x$-location of one ship frame are presented consistently in the time domain. The results for each frame are shifted in the time domain in such a way that the rising undisturbed wave crosses the still water level at the time instant $t=0 \mathrm{~s}$. The wave elevation for this is obtained from on the longitudinal cross-section FS (Fig. 3).

The pressure histories have been made non-dimensional with the properties of the encountered waves: an average encounter period and an average wave height. The averages have been calculated using the information on these wave properties on the cross-section FS (Fig. 3) between the $x$-location of the ship fore perpendicular and $x=5.86 \mathrm{~m}$ (see Fig. 2). The properties of the waves were analyzed from the time histories of the waves that were low-pass filtered with the threshold frequency of $3.5 \times$ encounter frequency.

Further, the average pressure is subtracted from the pressure signals in the case of constantly immersed sensors.

\section{Experimental approach}

\subsection{Instrumentation}

The model tests were performed in the towing tank of the Department of Applied Mechanics of Aalto University; see Table 7. The tank has a plunger-type wave maker at one end and, for damping the waves, beaches at the other end. In order to minimise the wave reflection, floats were positioned at the beach end of the tank during these experiments. The movement of the wave maker was controlled with a sine signal.

Table 7 Characteristics of the towing tank and the wave maker

\begin{tabular}{ll}
\hline Tank length & $130 \mathrm{~m}$ \\
Tank depth & $5.5 \mathrm{~m}$ \\
Tank breadth & $11 \mathrm{~m}$ \\
\hline
\end{tabular}

Plunger type wave maker with wedge inclination of $35^{\circ}$
The characteristics of the ship model are given in Table 1. The model was free to heave and pitch. The position of the model was balanced with weights in such a way that it was on an even keel with the selected model velocity. The draught given in Table 1 is the draught at the model velocity.

The measured data consist of the local pressures, wave height, model velocity, and heave movement of the model. The local pressures were measured using 10 pressure sensors (Kyowa's PGM-02KG) on the port side of the bow area; see Fig. 2. The sensors have a sensing diaphragm diameter of $12 \mathrm{~mm}$ [24]. Their rated capacity is $20 \mathrm{kPa}$ and the natural frequency is $2 \mathrm{kHz}$. The wave height was measured with a servo-mechanical wave height meter (Denshi Kogyo Co.'s VC-503). It was attached to the carriage $1.13 \mathrm{~m}$ in front of the fore perpendicular of the model and $0.15 \mathrm{~m}$ to port from the symmetry line. The velocity of the model was measured with a pulse encoder. The heave was measured near the aft and fore perpendiculars of the model with potentiometers to ensure that the ship motions were minor in the present wave conditions.

All the measured data were collected into a laptop via a National Instrument SCXI-1000 data acquisition box. The data acquisition box had one SCXI-1102B input module with an SCXI-1303 terminal block and another SCXI-1520 input module with an SCXI-1314 terminal block. The output signals of the pressure sensors were amplified with a DC amplifier before the sensors were connected to a terminal block. The sampling frequency was $1007 \mathrm{~Hz}$.

\subsection{On the calibration of the pressure sensors}

The calibration coefficients of the pressure sensors were determined with a specific calibration measurement in the towing tank. During a calibration, the model forward speed was zero and the hydrostatic pressure on the pressure sensors was altered. The responses of the pressure sensors were measured at six fixed levels of the hydrostatic pressure, which had a pressure difference of the water height of $2 \mathrm{~cm}$ between the adjacent levels. In order to alter the hydrostatic pressure, the vertical position of the ship model was changed with the adjustable towing rig of the carriage. Before a calibration measurement, the ship model was pressed downwards so that all the pressure sensors were in water.

Figure 4 shows an example of the calibration response in the case of the sensor at the location 4 (below the design water line) and in the case of the sensor 6 (above the design water line). The sensors above the design water line were pressed under the water just before starting the calibration measurement. The calibration measurement of the example in Fig. 4 includes measuring the responses both when pressing the ship model deeper into the water and both 

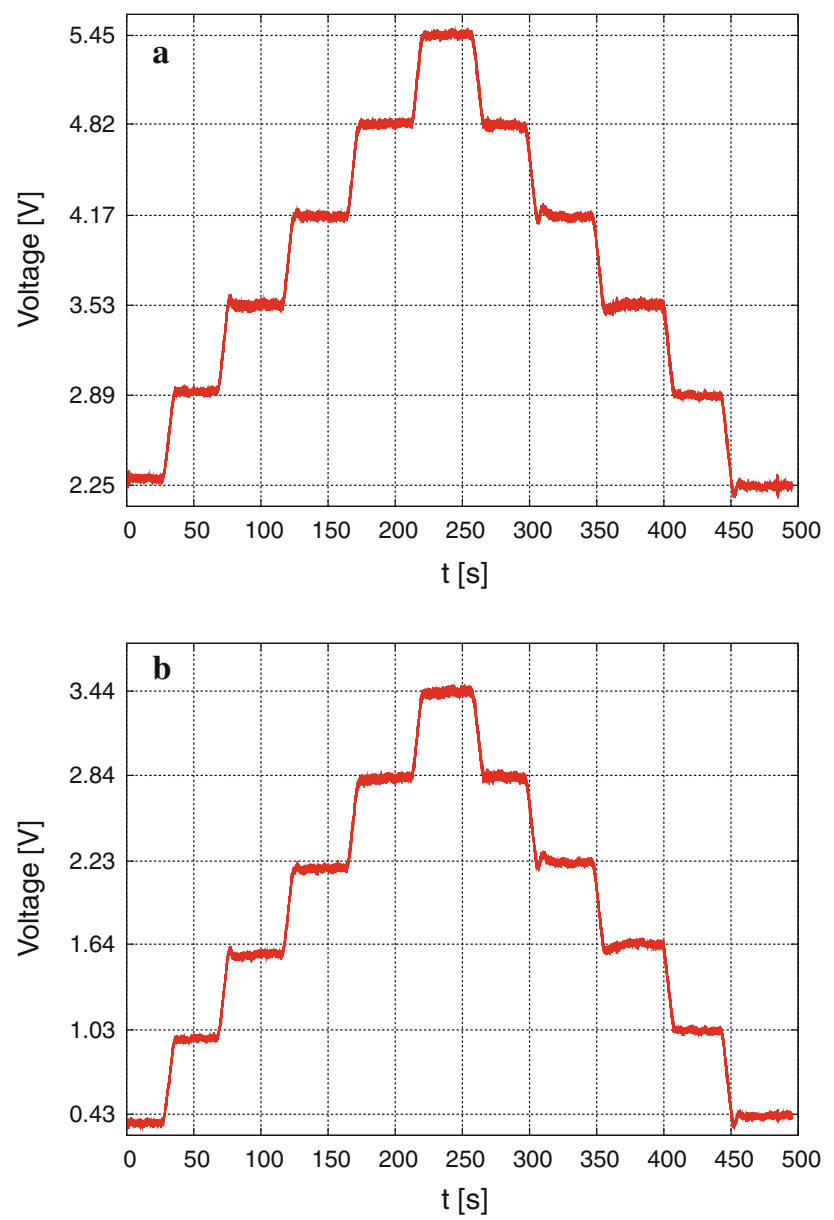

Fig. 4 Responses of a calibration measurement. a Sensor 4 (locates below design water line). b Sensor 6 (locates above design water line)

when lifting it back upwards. The response of the sensor above the design water line has larger differences between the downward $(0 \mathrm{~s}<t<220 \mathrm{~s})$ and upward $(260 \mathrm{~s}<t<500 \mathrm{~s})$ movement compared with a sensor below the design water line. This indicates that the response of the pressure sensors depends slightly on the time that they have been in water.

The calibration measurement was repeated several times in order to test the repeatability of the sensor behaviour. Some of the calibration measurements were done both when pressing down and lifting up the model and some of them were done only when pressing down the model. It was found out that the accuracy of the calibration coefficients was of a magnitude of $1 \%$ for each sensor. Further, the linearity of the pressure sensors was good, the coefficients of determination being larger than 0.998 for each calibration of each sensor. These numerical values indicate that the effect of the time that the sensors had been in water (see the example above) is very minor in terms of the calibration coefficients.
Measuring the zero levels of the pressure sensors was done without a forward speed.

\subsection{Representing the results on local ship wave loads}

The flow case was measured several times in order to have a representative sample of the waves that were encountered. As these steep waves are difficult to reproduce reliably over the length of the tank, the encounters corresponding to the target wave characteristics were selected afterwards. The selection criterion was that the wave height was within roughly $\pm 10 \%$ of the target wave height.

The purpose of the signal post-processing is to be able to compare the results of distinct wave encounters easily with each other and with the computed results.

The pressure signals are low-pass filtered with the threshold frequency of $30.5 \times$ encounter frequency as in the case of the computed results.

In practice, the selected data are from eight different runs and include 4-15 consecutive wave encounters from one run. The selected results from one run are treated separately before presenting them all together.

First, the average levels of the pressure signals are adjusted. In the case of the constantly immersed sensors, the mean value of the pressure is subtracted from the signal. In the case of the locations above the design water level, the signals are adjusted so that the air pressure gets the value zero.

Second, the pressure signals are synchronised with the signals of the wave elevation. As in the case of the computed results, the basic idea is that the instant when the rising undisturbed wave crosses the still water level is moved to the value $t=0 \mathrm{~s}$. In the case of the experimental results, this is done using the average encounter period of one run so that the selected wave encounters of one run fulfill this condition on average.

The selected pressure signals from one run are made non-dimensional with the respective average encounter period and average wave height.

\section{On the wave conditions}

The present computations include two assumptions that relate to the wave conditions. Firstly, it was assumed that the ship position can be fixed in the computations due to very short waves. Secondly, it was assumed that the waves can be generated with a numerical wave boundary condition that is based on the first order Stokes waves. In this section, the justifications of these assumptions are studied.

The measurement of the heave in the model tests revealed that the heave and the pitch motions are insignificant. The standard deviation of the pitch was less than 
$0.004 \mathrm{deg}$. The standard deviation of the heave was less than 0.003 and $0.006 \%$ of the ship length at the fore and aft perpendiculars, respectively.

Figure 5 shows a comparison of the encountered waves in the computations and in the model tests. The computational results are from the same $x$-location as the pressure sensors on the longitudinal cross-section FS (Fig. 3), while the experimental results are from the wave height meter in front of the bow and include all analysed wave encounters. The shapes of the time histories in the computations and in the model tests are in a reasonable agreement. Both the computational and the experimental waves are so-called Stokes waves with distinct non-linear features. Further, both of the results show that the shape of the waves varies. Comparing the computed waves between the three $x$-locations shows that the wave height varies as a function of the $x$-location too.

In the computations, the applied boundary condition creates higher harmonic waves that travel more slowly than the main wave. This results in oscillations of the wave height in the computational domain as a distance to the wave boundary. In the area of the ship bow, the wave height varies as a function of $x$ between 97 and $120 \%$ of the input-value of the wave height. The standard deviation of the average wave height for different encounters is less than $1 \%$ in the area of the bow. The encounter periods are nearly constant and slightly larger than the input-value. The standard deviations of the encounter periods for different encounters are even smaller than those of the average wave heights. Thus, the wave conditions are fixed as a function of time at a constant distance to the wave boundary, but they vary between different distances to the wave boundary.

Similarly to the computations, generating monochromatic waves is difficult in the model tests (see e.g. [25, 26]), because the movement of the wave maker does not correspond to the movement of the water particles.
Further, these steep waves tend to deform when they propagate. As a consequence of these two facts, each encountered wave in the model tests is different. The measured data include 63 encounters, which fulfil the criterion of the wave height being within roughly $\pm 10 \%$ from the target value. The statistical analysis of these selected data reveals that the encounter periods are slightly larger $(3 \%)$ than the target value and almost constant (standard deviation of $2 \%$ of the average). The wave height is, on average, very close to the target value, being $0.5 \%$ smaller, but it varies notably, with a standard deviation of $6 \%$.

The statistical data show that the waves are higher and steeper in the computations than in the model tests. This indicates that normalising the results is reasonable for the sake of the reasonable comparison between the computations and the model tests. In this context, it is relevant to understand that the relative distance between the ship model and the wave generator behaves differently between the two approaches. In the model tests, the ship model moves towards the wave generator and passes the wave field that is measured in front of the bow of the ship. In this respect, it is logical to use the information of the wave probe in front of the bow in order to normalise the experimental results. In the computations, the distance between the ship model and the wave boundary is fixed. Then, the wave conditions at a location, which has a similar distance to the wave boundary as the area of interest on the hull, should be used for the normalisation of the results. Because the deformation of waves on the hull may have a downstream effect, the computational results are normalised as explained in Sect. 3.3. The applied average wave heights are $0.090 \mathrm{~m}$ (coarse), $0.088 \mathrm{~m}$ (medium) and $0.086 \mathrm{~m}$ (fine).

Figure 6 compares the free-surface level near the hull between the experimental and computational results. Both approaches predict qualitative similar behaviour.
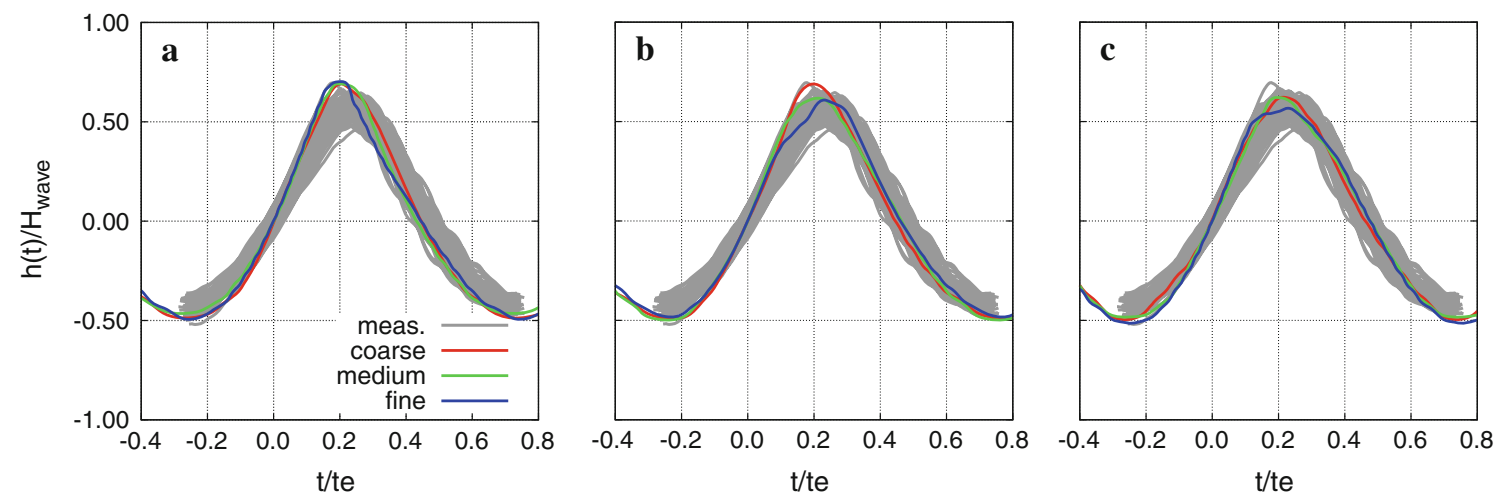

Fig. 5 Wave histories in the computations and in the model tests. The subfigures give the computed results at different locations. a $x=5.86 \mathrm{~m}$ b $x=6.04 \mathrm{~m}$ c $x=6.22 \mathrm{~m}$ 
Fig. 6 Free-surface behaviour in the area of the bow. Left computation with fine resolution. Right Model tests
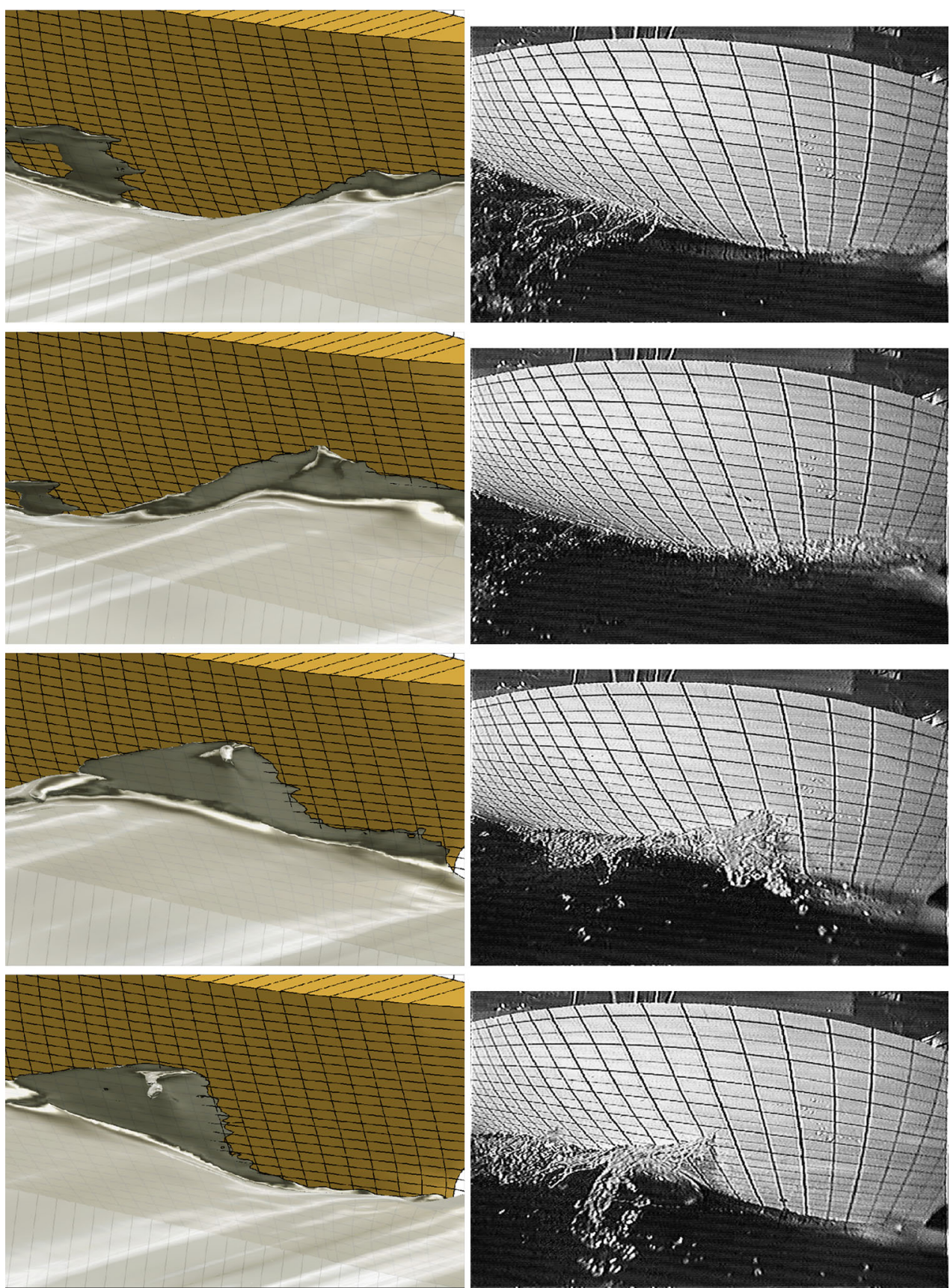

\section{Local wave loads}

Figure 7 gives an overall idea of the flow case on the basis of the computational results for the same view that was used in Fig. 6. It includes a representative sample of instant piezometric pressure distributions on the area of the bow in order to show how an important pressure impact propagates in the area of the bow. First, a large pressure impact occurs close to the ship fore perpendicular. Then, the pressure impact travels downstream together with the front of the encountered wave. The amplitude of this impact decreases when it travels further downstream. The pressure sensors are located behind the largest pressure values.

Next, the computed results are first presented with details on the solution behaviour. Then the computed and the measured results are compared.

The solution behaviour of the computed results was observed by studying both the effect of the iteration number and the effect of the resolution. It was found out that the effect of the iteration number on the results is minor from the practical point of view. In order to illustrate the largest effect of the iteration number on the pressure 

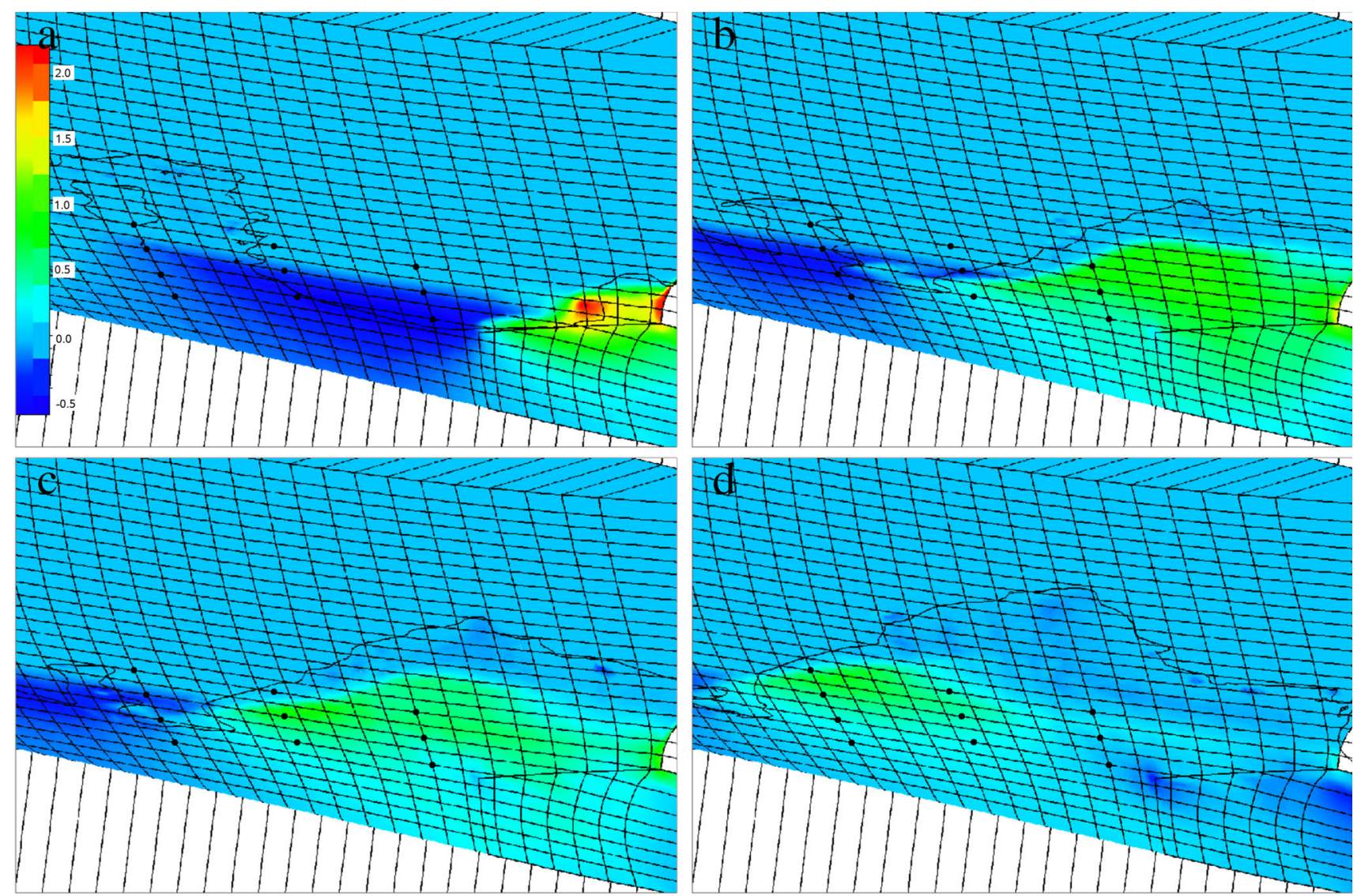

Fig. 7 Instant pressure distributions on the area of the bow. Non-dimensional piezometric pressure. Computation with fine resolution

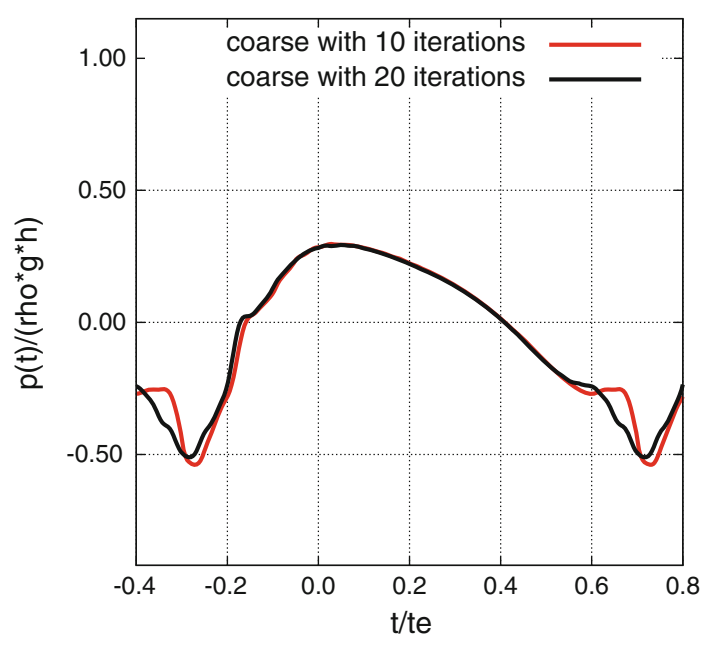

Fig. 8 An example of the iterative error. Sensor 4. Coarse resolution with 10 and 20 iterations

histories, the pressure histories with the two iteration numbers at the sensor 4 are compared in Fig. 8. The time span between the instants $t / t_{\mathrm{e}} \approx 0.6$ and $t / t_{\mathrm{e}} \approx 0.7$ shows the largest observed difference that the iteration number has on the results. As the effect of the iteration number on the results is minor from the practical point of view, only the results with 20 iterations are presented in the following.

Figure 9 gives the average pressure histories for the ten sensor locations with the three resolutions. In general, the three results look alike at each sensor, even if some local and instant differences exist.

Before analysing the agreement of the computational results with the different resolutions in more detail, it is necessary to underline that both the number and the locations of the computational points are different with the different resolutions. These differences affect the agreement of the results between the resolutions. Thus, analysing the effect of the resolution purely is not possible. As one example of the effect of the locations of the computational points, Fig. 10 gives the pressure histories for the distinct computational points of the fine resolution at the sensor 3. This example shows that the maximum value of the pressure can be very sensitive to the location of the computational points. Such sensitivity is typical for the sensors on the two uppermost rows. The largest effect is shown in this example. Another example (Fig. 10) is the behaviour of the distinct pressure histories of the medium resolution at the sensor 4 . In this case, both of the pressure histories show a spike at the instant $t / t_{\mathrm{e}} \approx 0.7$. However, its importance is much more 

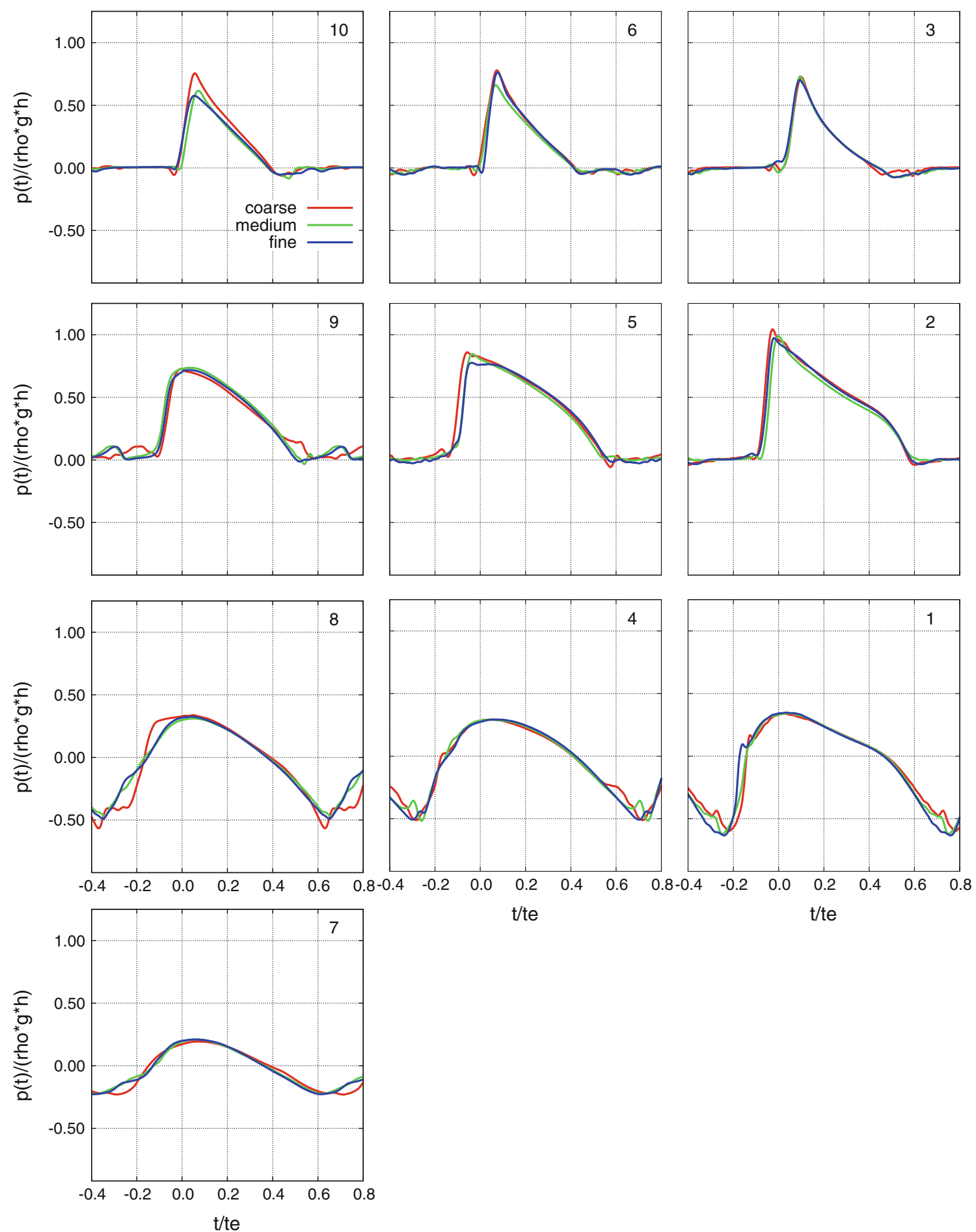

Fig. 9 Computed pressure histories with 20 iterations

significant according to Point 01 than according to Point 02. This second example illustrates that the importance, which a detail in the flow solution gets, can depend both on the locations and, as the average pressure histories of the computational points within a sensor are presented, on the number of the computational points within a sensor. Even if the results within the surface area of a sensor included several details, calculating an average from well-distributed computational points would smooth the solution.

As a further detail, the results in Fig. 10 include all the ten encounter periods for each distinct computational point with one colour. The results of the ten encounter periods 

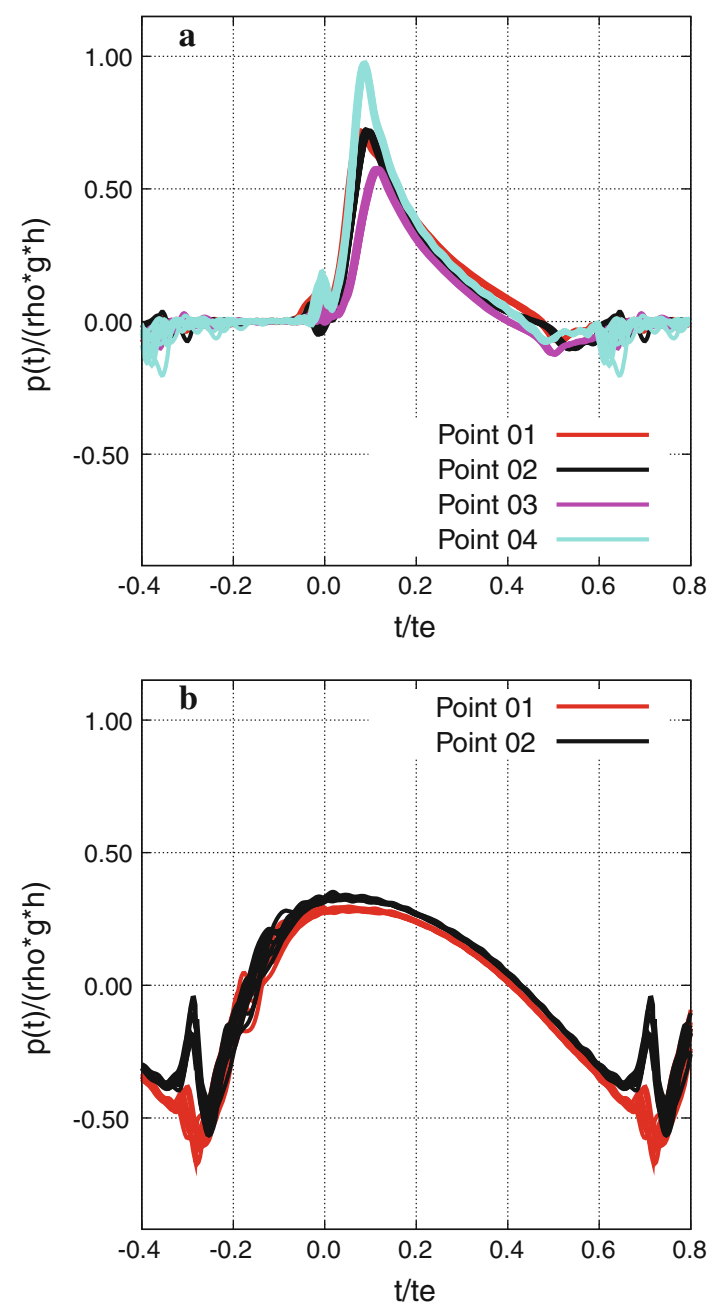

Fig. 10 Examples of the effect of the location of the computational points within the surface area of a pressure sensor. a Fine resolution with 20 iterations, sensor 3. b Medium resolution with 20 iterations, sensor 4

are very close to each other. This indicates an excellent repeatability of the encounters in the computations.

Next, the results in Fig. 9 can be analysed more closely when understanding that the difference between the resolutions relates both to the effect of the resolution and to the effect of the locations of the computational points. Above the design water line (sensors 10, 6, 3, 9, 5, 2), the shapes of the time histories at each sensor look alike between the three resolutions. To be more precise, the time histories have similar rises and descends in terms of both the length of the time and the shape of the time history. The main differences relate to the maximum peak values and the locations of these peaks in the time domain. In this regard, the results of the sensor 10 show the largest variation and the results of the sensors 3 and
9 the best agreement. Further, the behaviour of the pressure history close to the zero value can depend on the resolution. In this regard, the results of the sensor 9 show the largest variation.

Below the design water line (sensor 8, 4, 1, 7), the results of the three resolutions are fairly similar regarding both the amplitude and the shape of the time history in general. At a more detailed level, the shapes of the time histories of the fine and medium resolutions are more similar than the shapes given by the course resolution. This observation is the most distinct in the case of the sensors 7 and 8. Such a finding can indicate the convergence of the solution with the refinements of the resolution. On the other hand, the observation can indicate that these results are especially dependent on the distribution of the computational points within the surface area of a sensor.

The conclusion of the refinement study is that the general agreement of the results between the resolutions is good. The most distinct differences between the resolutions indicate an uncertainty that is locally and instantly larger.

Figure 11 shows that the computed pressure histories are in good agreement with the measured ones. Both the computational and the experimental results show similar differences between the pressure histories at the ten locations that were observed. The results of the two approaches are similar in the case of both impact-type and smoother behaviours: see, for example, location 3 versus location 7 . Further, the forms of the pressure histories are alike regarding both the rise and the fall of the histories at each location. The most visible difference between the computed and the measured pressure histories above the design water line (sensors 10, 6, 3, 9, 5, 2) is that the descent of the pressure levels to the zero level happens slightly faster in the case of the computed results. Below the design water line, the rise time of the sensor one is possibly shorter in the computations than in the model tests. Nevertheless, both the approaches capture a similar development of the local loading in the bow area observed.

Overall, the computed results show an excellent qualitative agreement with the measured ones. This encourages their further exploitation. Being able to capture the local load behaviour for the entire encounter period enables the development of the global loading on the bow area of the ship to be studied as a function of time. On that basis it is possible to learn the location of the critical area for the development of the global loading and to point out the dominant flow phenomena in the vicinity of that area. 

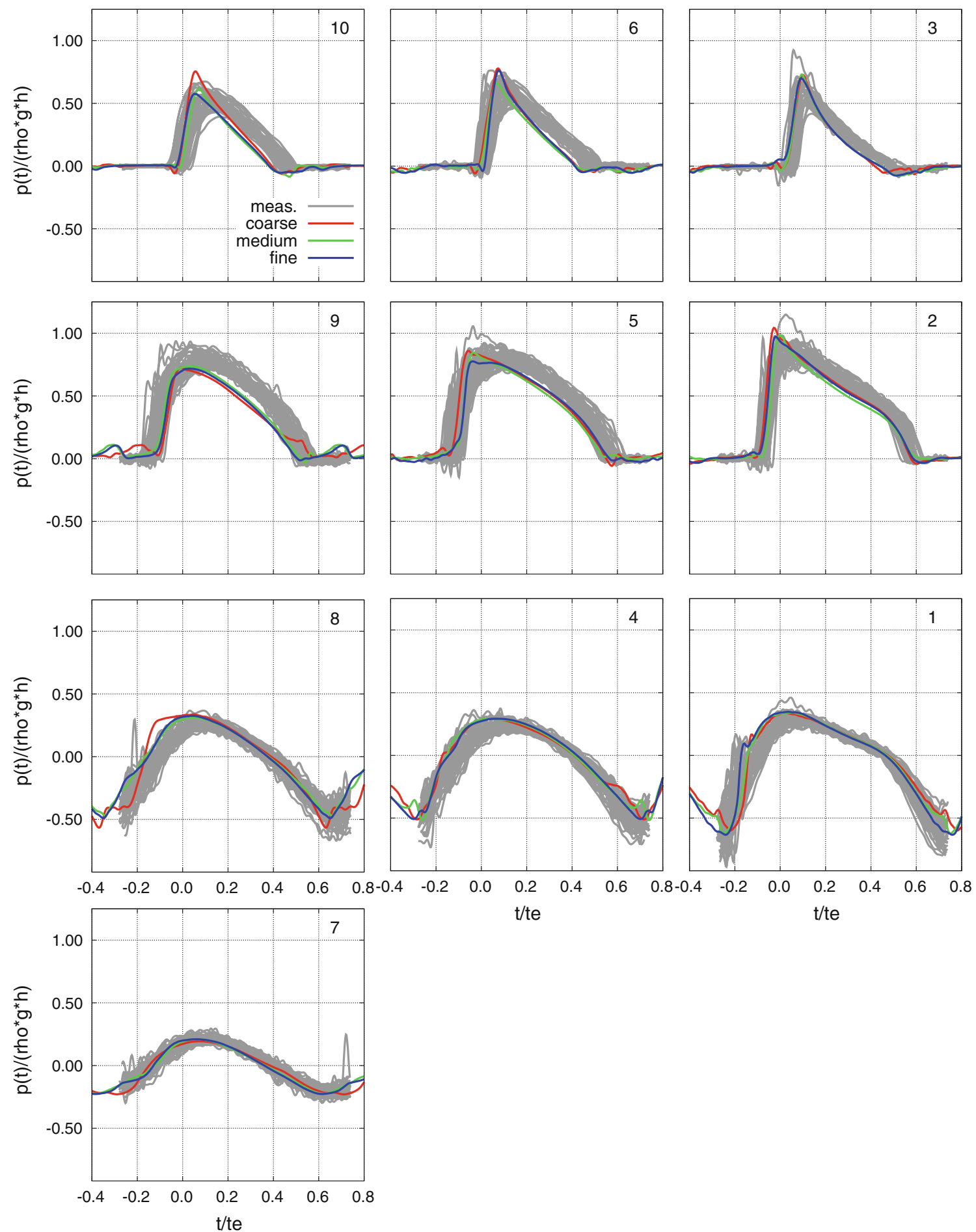

Fig. 11 Computed and measured pressure histories

\section{Conclusions}

This study considers the capability of an interfacecapturing method to predict local ship wave loads in short and steep waves by comparing its results with the experimental ones. The main focus is on the local pressure histories at ten locations on the bow of a ship.

It was shown qualitatively that the solution behaviour of the computed results is reasonable, even though the results can depend on the location of the computational points within the surface area of a pressure sensor. 
The agreement of the computed and the measured results is good at all the ten locations on the bow of the ship. The characteristics of the wave loads vary between the sensor locations. Both impact-type and smoother behaviours of the loading are captured well by the numerical method.

The present waves are very steep. As a consequence, they deform with quite a large range of variation when propagating. The present results show that this does not affect the qualitative behaviour of the local wave loads significantly. This study shows that the present computational results are adequate for further analysis of the flow case.

On a general level, this study shows that an interfacecapturing method can give qualitatively correct results in the case of very steep and short waves too when the discretisation resolutions, for instance, are carefully selected. Thus, this study increases confidence in the capability of such methods to predict ship wave loads.

Acknowledgments This study was carried out within a research project funded by the Academy of Finland. The financial support is gratefully acknowledged. The computational resources provided by CSC - the Finnish IT Center for Science-are also gratefully acknowledged. The authors are grateful to Laboratory Manager Keijo Hanhirova and the laboratory personnel of the Marine Technology Unit of Aalto University for helping to arrange the model-tests. The authors are grateful to Prof. Michel Visonneau and the CFD-team of ECN-CNRS for the discussions and the development of ISIS-CFD.

Open Access This article is distributed under the terms of the Creative Commons Attribution License which permits any use, distribution, and reproduction in any medium, provided the original author(s) and the source are credited.

\section{References}

1. Sato Y, Miyata H, Sato T (1999) CFD simulation of 3-dimensional motion of a ship in waves: application to an advancing ship in regular heading waves. J Mar Sci Technol 4:108-116

2. Orihara H, Miyata H (2003) Evaluation of added resistance in regular incident waves by computational fluid dynamics motion simulation using an overlapping grid system. J Mar Sci Technol $8: 47-60$

3. Hino T (ed) (2005) The Proceedings of CFD Workshop Tokyo 2005. National Maritime Research Institute, Tokyo

4. Klemt M (2005) RANSE simulation of ship seakeeping using overlapping grids. Ship Technol Res 52:65-81

5. Carrica PM, Wilson RV, Stern F (2006) Unsteady RANS simulation of the ship forward speed diffraction problem. Comput Fluids 35:545-570

6. Carrica PM, Wilson RV, Noack RW, Stern F (2007) Ship motions using single-phase level set with dynamic overset grids. Comput Fluids 36:1415-1433
7. Oberhagemann J, el Moctar O, Schellin TE (2008) Fluid-structure coupling to assess whipping effects on global loads of a large containership. In: Proceedings of 27th Symposium on Naval Hydrodynamics, Seoul, Korea

8. Visonneau M, Queutey P, Leoroyer A, Deng GB, Guilmineau E (2008) Ship motions in moderate and steep wave with an interface capturing method. In: Proceedings of 8th International Conference on Hydrodynamics, Nantes, France, pp 485-491

9. Zwart PJ, Godin PG, Penrose J, Rhee SH (2008) Simulation of unsteady free-surface flow around a ship hull using a fully coupled multi-phase flow method. J Mar Sci Technol 13:346-355

10. Deng GB, Queutey P, Visonneau M (2009) Seakeeping prediction for a container ship with RANS computation. In: 22nd Chinese Hydrodynamic Conference, Chengdu, China

11. Larsson L, Stern F, Visonneau M (eds) (2010) A workshop on Numerical Ship Hydrodynamics, Proceedings, vol II, Gothenburg, Sweden

12. Oberhagemann J, Holtmann M, el Moctar O, Schellin TE, Kim D (2009) Stern slamming of a LNG carrier. J Offshore Mech Arct Eng 131

13. Carrica PM, Fu H, Stern F (2011) Computations of self-propulsion free to sink and trim and of motions in head waves of the KRISO Container Ship (KCS) model. Appl Ocean Res 33:309-320

14. Castiglione T, Stern F, Bova S, Kandasamy M (2011) Numerical investigation of the seakeeping behaviour of a catamaran advancing in regular head waves. Ocean Eng 38:1288-1806

15. Orihara H (2011) Comparison of CFD simulations with experimental data for a tanker model advancing in waves. Int $\mathbf{J}$ Nav Archit Ocean Eng 3:1-8

16. Guo BJ, Steen S, Deng GB (2012) Seakeeping prediction of KVLCC2 in head waves with RANS. Appl Ocean Res 35:56-67

17. Hänninen SK, Mikkola T, Matusiak J (2012) On the numerical accuracy of the wave load distribution on a ship advancing in short and steep waves. J Mar Sci Technol 17:125-138

18. Hänninen SK, Mikkola T (2008) Wave excitation on a ship bow in short waves. In: Proceedings of 11th Numerical Towing Tank Symposium, Brest, France

19. Hänninen S, Mikkola T, Matusiak J (2011) Comparing computed and measured ship wave load distributions. In: Proceedings of 14th Numerical Towing Tank Symposium, Poole, England

20. Queutey P, Visonneau M (2007) An interface-capturing method for free-surface hydrodynamic flows. Comput Fluids 36:1481-1510

21. Numeca International (2011) Theoretical Manual FINE ${ }^{\mathrm{TM}} / \mathrm{Mar}-$ ine $\mathrm{V} 2.3$

22. Wackers J, Koren B, Raven HC, van der Ploeg A, Starke AR, Deng GB, Queutey P, Visonneau M, Hino T, Ohashi K (2011) Free-surface viscous flow solution methods for ship hydrodynamics. Arch Comput Methods Eng 18:1-41

23. Numeca International (2011) User manual FINE ${ }^{\mathrm{TM}} / \mathrm{Marine} \mathrm{v} 2.3$, Documentation v2.3b

24. Kyowa. Product information. Available at http://www.kyowa-ei. co.jp/eng/product/sensors/pressure/pgm_g.html

25. Henderson DM, Patterson MS, Segur H (2006) On the laboratory generation of two-dimensional, progressive, surface waves of nearly permanent form on deep water. J Fluid Mech 559:413-427

26. Mikkola T (2006) Time accurate simulation of a plunger type wave maker using unstructured finite volume solver with surface tracking. In: Proceedings of 26th Symposium on Naval Hydrodynamics, Rome, Italy 